Nous sommes donc en présence d'une méthode très sûre, un peu délicate sans doute, mais remplissant toutes les conditions exigées, c'est-à-dire capable de doser les radicaux lactiques avec certitude, malgré la présence de lactose et de matières organiques azotées.

Nous allons maintenant l'appliquer à l'étude des macérations aqueuses de caséine lactique.

\title{
BIBLIOGRAPHIE.
}

(A suivre.)

[1] Maheu. Thèse Fac. Méd., Paris 1921. Lefrançois.

[2] DentGìs. Traité Chimie analytique.

[3] Suzuki et Hart. Journ. of Am. Chem. Soc., XXXI, p. 1364, 1909.

[4] Simon et Praut. Bull. Soc. Chim. Biol., 1924, p. 477.

[5] E. Jerusalem. Bioch. Zeits., 1908, p. 361 et 379.

[6] Sobolewa et Zaleski. Zeits. Phys. Chem., CXIX, p. 441, 1910.

[7] Bellet. Bull. Soc. Chim. Fr., XIII, p. 565, 1913.

[8] Claudsen. Cité par Densgès, Traité chimie analytique.

[9] Chelle. Bull. Soc. Phys., Bordeaux 1914.

[10] TAPernoux et Ferry, C. R. Soc. Biol., 1929, no 14, p. 1213.

\section{A PROPOS D'UNE NOUVELLE IMÉTHODE DE NUMÉRATION DIRECTE DES MICROBES DU LAIT}

\author{
par M. Emm. POZZI-ESCOT
}

Professeur de Chimie à l'Institut National Agronomique du Pérou, Fondateur du Laboratoire national de microbiologie agricole du Pérou.

Le Lait nous donne dans le numéro de janvier de cette année (page 37 et suivantes) une étude très intéressante de M. BoGDANOFF sur une nouvelle méthode de numération directe des microbes du lait, pour laquelle il utilise le mélange du lait avec une émulsion de concentration connue de cellules volumineuses (levures Schizo). Sans méconnaître les mérites que peut présenter ce procédé dans certains cas particuliers (les levures Schizo sont surtout communes dans nos pays chauds), je désire cependant signaler aux lecteurs de la revue Le Lait qu'il y a de bien longues années que j'utilise un procédé en tous points analogue et que je l'ai fait connaître dans toute une série de publications, qui, je le vois, n'ont pas mérité de retenir l'attention du nouvel inventeur, et, cependant, le Bulletin de la Société Chimique de Belgique, de la Société Chimique de France, Le Lait lui-même et Chemical Abstracts sont des références qui, d'habitude, suffisent amplement.

Je dois reconnaître qu'à l'époque de ma publication, la question de la numération bactérienne dans le lait, appliquée depuis bien longtemps déjà en Amérique, était ou tout à fait inconnue en Europe ou 
seulement connue de quelques rares spécialistes. J'ai enseigné, dans mes cours de contrôle laitier, la méthode de Breed, puis celle de Frost, il y a déjà plus de 20 ans ; et c'est en voyant qu'on commençait à s'intéresser à elles en Europe que j'ai fait connaître ma méthode rapide de numération microbienne applicable au contrôle industriel des laits, par des publications faites en 1922. On en trouvera la relation aux Annales et Revue de Chimie analytique de 1922; au Bulletin de la Société Chimique de Belgique de la même année; au Bulletin de la Société Chimique de France, t. XXXVI, p. 159; dans Le Lait, qui a publié un extrait, t. IV, p. 238.

J'ai signalé à l'époque qu'il n'y a dans mon procédé et, par suite, également dans celui de BOGDANOFF, qu'une application d'un proeédé d'usage courant dans les laboratoires de microbiologie pour la détermination de la force des émulsions vaccinales ; e'est en somme la méthode de Wright, dont on peut varier à son goût le mode opératoire principe. Certainement que le mode opératoire dont j'ai indiqué il y a 10 à 12 ans le principe dans les publications que je viens de rappeler a été considérablement amélioré à l'usage. Si les chimistes de laiterie étaient moins spécialistes, il est évident qu'ils auraient reconnu dès longtemps les avantages de cette manière de faire.

Le procédé de Wright pour l'évaluation de la force des vaccins consiste à mélanger à $1 \mathrm{~cm}^{3}$ d'émulsion vaccinale dans de l'eau physiologique colorée avec du bleu de méthylène, un égal volume de sang humain et $3 \mathrm{~cm}^{3}$ d'eau physiologique. On prélève une goutte de ce mélange qu'on étale sur une lame porte-objet et qu'on laisse sécher; à l'examen, on compte, d'une part, les hématies et, d'autre part, les éléments microbiens. Il ne reste plus qu'à établir le rapport des uns aux autres pour en déduire le nombre de microbes dans le vaccin. Dans le cas du lait, où les microbes sont moins nombreux, j'ai simplement indiqué d'user de sang dilué.

Il faut reconnaître que bien peu de chimistes s'occupant du contrôle laitier sont microbiologistes et que nos microbiologistes n'entendent rien, d'habitude, au contrôle laitier, ce qui explique bien qu'il ait fallu attendre si longtemps la généralisation d'un procédé tellement plus simple que la méthode de numération directe de Breed et Brew, puisqu'elle supprime totalement la difficile question des aires et des microvolumes.

On m'excusera d'avoir rappelé mon rôle dans cette généralisation : le Pérou est tellement en dehors de la sphère des relations courantes qu'on oublie véritablement trop souvent ce qui nous appartient.

Lima (Pérou).

(Institut des Hautes Etudes agronomiques, janvier 1934.) 